\title{
Clinical Experience with Nimotuzumab in Cuban Pediatric Patients with Brain Tumors, 2005 to 2007
}

\author{
Giselle Saurez, MD, Ricardo Cabanas, MD, Melba Zaldívar, MD, Tania Garnier, MD, Bárbara Iglesias, MD, Patricia Piedra, MD, \\ Martha Ríos Castillo, MD, Martha Longchong, MD, PhD, Normando Iznaga, PhD, Agustín Lage, MD, PhD
}

\begin{abstract}
Introduction Nimotuzumab, developed in Cuba, is a humanized monoclonal antibody that targets the epidermal growth factor receptor (EGFR). It has been evaluated in malignant brain tumors in adults and children, and shown to be therapeutically safe and effective in terms of increased survival and improved quality of life.
\end{abstract}

Objective Describe nimotuzumab's safety profile and clinical benefits in terms of disease control and survival in pediatric patients with progressive or recurrent primary brain tumors who were included in an expanded access program.

Methods An open, prospective clinical study was designed. Between December 2005 and December 2007, 22 patients were included, all of whom had an histological and/or radiological diagnosis of progressive or recurrent primary brain tumor, classified as high-grade malignant glioblastoma $(n=6)$, diffuse brain stem glioma $(n=6)$, ependymoblastoma $(n=5)$, low-grade glioma $(n=4)$, or thalamic tumor $(n=1)$; life expectancy of at least 4 weeks; and a Karnofsky or Lansky Performance Status score of $\geq 50$. Nimotuzumab was administered on a $100 \mathrm{mg}$ weekly intravenous infusion schedule for 6 to 8 weeks, followed by a bi-weekly maintenance phase, as long as there was no deterioration in the patient's functional capacity. Therapeutic protocols were

\section{INTRODUCTION}

As a group, malignant tumors of the central nervous system (CNS) are the most frequent solid tumors in children and adolescents, and the second most common type of malignant disease in these age groups. These tumors include a heterogeneous group of histological subtypes, predominantly of glial origin.[1,2]

Incidence of this type of tumor in Europe and North America is 3 per 100,000 population aged <15 years. [3] In Cuba, about 30 cases are reported annually for a rate of 1.3 per 100,000 population aged $<16$ years. [4]

High-grade gliomas account for about $15 \%-20 \%$ of all pediatric CNS tumors, slightly more frequent in children aged 5-10 years. [5] They vary in histological characteristics and sites of origin, and are usually supratentorial in both cerebral hemispheres or in the brain stem, although they may be found in the cerebellum and spinal region.[6,7] This group is mainly made up of anaplastic astrocytoma, glioblastoma multiforme and mixed glial tumors with a predominance of malignant astrocytic elements.[5-7] Their malignant features are generally varied and may include hypercellularity, cell atypia, mitosis, necrosis, endothelial proliferation and other anaplastic elements. They are very invasive, with the worst prognosis corresponding to glioblastoma multiforme.[1,5]

Survival with these tumors varies, depending on factors such as the patient's age, tumor composition, disease stage (newly followed for administration as monotherapy or in combination with chemotherapy and/or radiotherapy. All patients received clinical and imaging follow-up.

Results Nimotuzumab was well tolerated in all therapeutic modalities, even with prolonged exposure. A minority of patients reported slight or moderate adverse events, such as vomiting, mucositis and chills, as classified by the Common Terminology Criteria for Adverse Events (CTCAE). The disease was controlled in $64 \%(14 / 22)$ of patients; 6 -month and 1 -year survival rates were $82 \%$ and $64 \%$, respectively; average survival was 20.3 months and median survival, 19 months. Recovery of neurological functions and improvement in general status were notable in patients who attained control of the disease.

Conclusions As used in this study, nimotuzumab demonstrated a broad safety profile, making it acceptable for chronic use, and implied clinical benefits in terms of increased survival and improved functional status in these patients, compared to findings described in the literature. These results indicate further studies of this product are warranted.

Keywords: Central nervous system neoplasms, brain neoplasms, brain cancer, biological therapy, immunotherapy, monoclonal antibodies, EGF receptor, child mortality, nimotuzumab.

diagnosed or recurrent), and treatment administered (involving surgery, radiotherapy and chemotherapy as a multimodal treatment).[8-12] In newly-diagnosed patients with low-grade glioma, chemotherapy (carboplatin and vincristine) has demonstrated a $70 \%$ disease-free survival rate.[12] In brain stem tumors, radiotherapy is the treatment of choice, and chemotherapy offers no added benefit. However, survival at 18-24 months after diagnosis is $\leq 10 \%$. [12]

In Cuba, a study on behavior of childhood brain tumors in a pediatric oncology service, conducted from January 1990 to December 2000, reported that more than half of 62 diagnosed and treated patients died less than a year after diagnosis, and only $35.5 \%$ were alive after 5 years.[4]

Therapeutic options for progressive or recurrent tumors are very limited. Despite scientific progress in developing new therapies, none has yet prolonged survival in these cases, which generally drops dramatically to as low as $3-4$ weeks.[11,12] Thus, the search for new therapies to improve survival is imperative. Several clinical trials are underway to evaluate such new therapies combined with those described above, seeking optimal therapeutic regimens capable of controlling the disease and improving patients' quality of life.

Innovative therapeutic strategies, known as targeted therapies, have been clinically evaluated,[13] including tyrosine kinase 
inhibitors and monoclonal antibodies targeting the epidermal growth factor and its receptor (EGF/EGFR). This receptor is highly overexpressed in brain tumors, which is why intervening in tumor proliferation mechanisms-blocking them and inhibiting intracellular signals that regulate angiogenesis, invasiveness and resistance to radio- and chemotherapy - may be a very attractive strategy.[14]

Nimotuzumab is a humanized monoclonal antibody that targets the EGFR, developed in Cuba by the Molecular Immunology Center (CIM, its Spanish acronym) and marketed by CIMAB. $[15,16]$ It is an IgG1 isotype immunoglobulin that binds with intermediate affinity to the EGFR, obtained through genetic engineering by transplanting the complementarity determining regions (CDR) in a human immunoglobulin framework.

In 2005, the Government Center for Quality Control of Medicines (CECMED, its Spanish acronym), the Cuban regulatory agency, licensed nimotuzumab for treatment of advanced head and neck tumors, and in 2007 extended the license to treatment of malignant glioma in adults and children.

This antibody has been evaluated in multiple clinical trials in Cuba[17-20] and other countries, including Germany,[21,22] Canada,[23,24] China,[25] United States, [26] India,[27-29] Japan and South Korea,[30] involving more than 1,000 patients with different types of epithelial-origin tumors, fundamentally advanced head and neck tumors and CNS tumors. In these studies, the product has been shown to be safe and effective in terms of disease control and prolonged survival.

However, clinical experience with nimotuzumab in pediatric patients is very limited. The only results available to us come from phase II and phase III studies carried out in Germany in patients with brain stem tumors and later published by the American Society of Clinical Oncology (ASCO).[21,22] Inspired by the evidence of safety and efficacy shown in those studies, in 2005 the CIM-CIMAB community of pediatric oncologists and scientists designed an ongoing phase II clinical trial in brain stem tumors in Cuba. At the same time, an expanded access program was developed for patients who did not meet the inclusion criteria for the clinical trial, but who were considered likely to benefit from treatment.

Expanded access programs using new products about to be licensed have been developed in recent years with the approval of the US Food and Drug Administration (FDA) and the European Medicines Agency (EMEA).[31-34] These programs are designed for patients with malignant disease, for whom standard treatment is not effective or viable, because resulting survival time would be marginal or because of extreme toxicity. However, access outside the clinical trial context-such as this type of program applying more flexible criteria for use of a new product-may have clinical benefits.

The objective of this study is to describe the Cuban experience using nimotuzumab in children, in terms of safety profile and therapeutic benefit, as a result of the expanded access program for pediatric patients with brain tumors in Cuban pediatric oncology services.

\section{METHODS}

An open, prospective clinical study was designed for pediatric patients with a diagnosis of progressive or recurrent primary brain tumor (confirmed by NMR/CAT or pathology results). This study covers all pediatric oncology services in Cuba, in coordination with the National Pediatric Oncology Program, and reported to CECMED.

General patient inclusion criteria were: age 3-18 years, life expectancy of at least 4 weeks, functional capacity $\geq 50$ on the Karnofsky or Lansky Performance Scale, and informed consent from parents or legal guardians. The study was approved by the Ethics Committees of participating institutions.

Data were collected on the 22 patients with progressive or recurrent primary brain tumors included in the study between December 2005 and December 2007 who received at least one dose of nimotuzumab.

Patients came from three hospitals: 16 from Juan Manuel Márquez Pediatric Teaching Hospital in Havana; four from Octavio de la Concepción y de la Pedraja Hospital in Holguín; one from the Pediatric Oncology Service of the National Oncology and Radiobiology Institute (INOR, its Spanish acronym) in Havana; and one from Pepe Portilla Pediatric Hospital in Pinar del Río.

Patients' baseline characteristics before receiving nimotuzumab treatment are shown in Table 1. Average age was 8 years (range 2-16 years), and the male/female ratio was 2:1 (15 male/7 female). Patients with recurrent high-grade malignant glioma (including anaplastic astrocytoma and glioblastoma multiforme) were most frequently represented, followed by those with diffuse brain stem gliomas and ependymoblastomas.

Less frequent were low-grade malignant glioma (including grade II fibroblastic astrocytoma and thalamic tumor). Most patients had been previously treated with surgery (for tumor resection or biopsy) followed by radiotherapy and/or radiochemotherapy regimens. Patients with brain stem or thalamic tumors for whom surgery was not an option had received only radiotherapy (one of them had received a suboptimal dose of 1026 cGy). All chemotherapy patients had received at least one line of treatment.

Treatment with nimotuzumab applied protocols using the monoclonal antibody as a single agent (monotherapy), in combination with radiotherapy, or with concurrent chemotherapy, in accordance with standard treatment.

For the induction phase, a uniform dose (independent of the patient's weight or height) of $100 \mathrm{mg}$ of nimotuzumab diluted in $250 \mathrm{ml}$ of sodium chloride solution was administered as a 30- to 60-minute intravenous infusion once a week for the first 6 weeks. An additional consolidation phase using the same dose administered every 2 weeks was also allowed, as long as there was no deterioration in the patient's functional capacity nor any severe adverse event or unmanageable intolerance to the drug.

Toxicity was evaluated by describing adverse events, identified by physical examination and laboratory tests carried out prior to treatment and monthly thereafter. Laboratory tests included hemoglobin and hematocrit, leukocyte and platelet counts, pyruvic and oxalacetic transaminases (ASAT/ALAT), alkaline phosphatase, creatinine and bilirubin. 
Table 1: Nimotuzumab Treatment Regimens and Patient Evolution

\begin{tabular}{|c|c|c|c|c|c|c|c|c|c|}
\hline \multicolumn{3}{|c|}{ Patient Baseline Characteristics } & \multirow{2}{*}{$\begin{array}{l}\text { Treatment } \\
\text { Regimen }\end{array}$} & \multirow{2}{*}{$\begin{array}{l}\text { Number } \\
\text { of Doses } \\
\text { Received }\end{array}$} & \multicolumn{2}{|c|}{ Safety Profile } & \multicolumn{3}{|c|}{ Clinical Evolution } \\
\hline Sex & Age & Prior Therapy & & & $\begin{array}{l}\text { Adverse } \\
\text { Event }\end{array}$ & Causality & $\begin{array}{l}\text { Best Clinical } \\
\text { Response }\end{array}$ & $\begin{array}{c}\text { Status at Last } \\
\text { Visit }\end{array}$ & $\begin{array}{l}\text { Survival } \\
\text { (months )* }\end{array}$ \\
\hline \multicolumn{10}{|c|}{ Diffuse Intrinsic Pontine Glioma (DIPG), 6 patients } \\
\hline M & & $\mathrm{RT}+\mathrm{CT}$ & $\begin{array}{l}\text { Comb. CT (CPM } \\
\text { and Procarbazine) }\end{array}$ & & 1 Hepatitis B & None & SD & Alive (SD) & 19.7 \\
\hline $\mathrm{F}$ & & RT & Monotherapy & & 0 Mucositis & Possible & SD & Alive (SD) & 19.9 \\
\hline M & & RT & Monotherapy & & $5 \begin{array}{l}\text { Vasovagal } \\
\text { reaction }\end{array}$ & Probable & SD & Alive (SD) & 22.5 \\
\hline M & & RT (only 1026 cGy) & $\begin{array}{l}\text { Comb. CT } \\
\text { (Cisplatin, CPM } \\
\text { and Procarbazine } \\
\text { + RT (4400 cGy) }\end{array}$ & & 9 Bone Pain & None & PR & Alive (PR) & 26.8 \\
\hline $\mathrm{F}$ & & $\begin{array}{l}\text { Surgery (partial resection) } \\
+ \text { RT + CT (Cisplatin and } \\
\text { CPM) }\end{array}$ & $\begin{array}{l}\text { Comb. CT } \\
\text { (Cisplatin and } \\
\text { CPM in 6th cycle) }\end{array}$ & & $\begin{array}{l}\text { ASAT/ALAT } \\
8 \text { alterations, chills } \\
\text { and seizures }\end{array}$ & $\begin{array}{l}\text { Possible } \\
\text { (only chills) }\end{array}$ & SD & Deceased (PD) & 18.8 \\
\hline F & & $\begin{array}{l}\text { Surgery }(90 \%)+C T \text { (2 lines) } \\
\text { (Cisplatin and CPM) }\end{array}$ & Monotherapy & & 2 None & None & SD & Alive (SD) & 13.5 \\
\hline M & & $\begin{array}{l}\text { Surgery + RT + CT (Cisplatin } \\
\text { and CPM) }\end{array}$ & Monotherapy & & 0 None & None & CR & Alive (CR) & 30.5 \\
\hline
\end{tabular}

\section{Anaplastic Astrocytoma, 3 patients}

Hospitalization

(Surgery),

Endocraneal Possible

F $\quad 14 \begin{aligned} & \text { Surgery ( } 3 \text { times })+ \\ & \text { RT }+ \text { CT (TMZ) }\end{aligned} \quad$ Monotherapy

Surgery (partial resection)

\section{M $\quad 13+\mathrm{RT}+\mathrm{CT}$ (Cisplatin and} CFM; TMZ)

\begin{tabular}{|c|c|c|c|c|c|c|c|}
\hline M & 8 Surgery $(95 \%)+R T$ & Monotherapy & 10 None & None & CR & Alive (CR) & 10.5 \\
\hline \multicolumn{8}{|c|}{ Gioblastoma Multiforme (GBM), 3 patients } \\
\hline M & 2 Surgery & $\begin{array}{l}\text { Comb. CT (VP-16 + } \\
\text { VCR + CPM) }\end{array}$ & 12 None & None & PD & Deceased (PD) & 5.7 \\
\hline M & 6 Surgery + RT & Monotherapy & 14 None & None & PD & Deceased (PD) & 6.9 \\
\hline $\mathrm{F}$ & 4 Surgery $(100 \%)+R T$ & Monotherapy & 20 None & None & PD & Deceased (PD) & 9.2 \\
\hline \multicolumn{8}{|c|}{ Low-Grade Glioma (LGG), 4 patients } \\
\hline $\mathrm{F}$ & 9 Surgery $(70 \%)+\mathrm{RT}$ & Monotherapy & 8 None & None & PD & Deceased (PD) & 14.7 \\
\hline $\mathrm{F}$ & $16 \begin{array}{l}\text { Surgery (partial } \\
\text { resection) + RT }\end{array}$ & $\begin{array}{l}\text { Comb. CT (VCR + } \\
\text { Carboplatin) }\end{array}$ & 17 None & None & SD & Alive (SD) & 14.7 \\
\hline M & 6 Surgery & $\begin{array}{l}\text { Comb. RT } \\
(6000 \text { cGy) }\end{array}$ & 28 None & None & SD & Alive (SD) & 26.4 \\
\hline M & $\begin{array}{l}\text { Surgery + } 2 \text { lines CT } \\
7 \text { (Cisplatin and CFM; } \\
\text { VCR and Carboplatin ) }\end{array}$ & Monotherapy & 55 None & None & SD & Alive (SD) & 33.1 \\
\hline \multicolumn{8}{|c|}{ Thalamic tumor, 1 patient } \\
\hline M & $8 \mathrm{RT}$ & Monotherapy & 8 None & None & PD & Deceased (PD) & 2.1 \\
\hline
\end{tabular}

${ }^{*}$ From date of first treatment until patient's death or last record of patient examination

CPM: Cyclophosphamide; CR: Complete response; CT: Chemotherapy; F: Female; M: Male; PD: Progressive disease; PR: Partial response; RT: Radiotherapy; SD: Stable disease; TMZ: Temazolamide; VCR: Vincristine; VP-16: Etoposide.

Source: Case Record Form and patient medical charts. 
Adverse events were classified by intensity according to the US National Cancer Institute's Common Terminology Criteria for Adverse Events (CTCAE) toxicity scale, version 3,[35] and by consequences for the patient; life-threatening and fatal events, as well as those requiring hospitalization, resulting in disability or invalidity, or producing teratogenic effects were classified as severe adverse events (SAE). In terms of causality, each adverse event was classified as probably, possibly, or not related to treatment with nimotuzumab.

Clinical evaluations consisted of clinical examination (evaluation of functional capacity and neurological functions) and imaging (CAT/NMR), before initiating nimotuzumab treatment and every 12 weeks after treatment. Results of the follow-up evaluations were compared with the initial evaluation to determine the objective clinical response in accordance with WHO's antitumor response classification.[36]

Disease control is defined as the best response obtained in the follow-up period, classified as complete response (tumor disappears), partial response (50\% decrease in tumor size), and stable disease or no evidence of progression (no change in tumor size).

Survival was measured as the number of months from the date of first treatment to the date of death or of the last programmed visit when information about the patient was available.

Clinical data were obtained from the Case Report Form (CRF) designed for this study and verified against primary data in individual medical charts. CRFs were monitored by the CIM/CIMAB Medical Board and the National Coordinating Center for Clinical Trials (CENCEC, its Spanish acronym). Clinical case discussion workshops were also held to validate information presented by the physician in charge of each case. A database was created with the clinical variables mentioned above. Statistical analysis was performed using the SPSS statistical package, version 11.5 for Windows. Descriptive statistics were used for quantitative variables (average, median) and percentages were calculated. The logrank test was used for survival analysis.

\section{RESULTS}

Since all patients had recurrent or progressive disease, administration of nimotuzumab alone was the only alternative in the majority of cases (15/22 patients). Five patients received combined treatment with second or third lines of chemotherapy; two patients received nimotuzumab combined with radiotherapy (one with radiotherapy alone and the other with the concurrent chemoradiotherapy combination). All received standard therapeutic doses and schedules defined for their disease in the institution where they were treated (Table 1).

Most of the patients (20/22) completed the nimotuzumab induction phase. In two cases treatment was interrupted before 6 weeks due to disease progression and early death.

Average number of doses received was 26 (range 1-60 doses), with exposure to nimotuzumab ranging between 0.4 and 30.1 months.

Safety Profile Adverse events during treatment were reported in 9 of the 22 patients treated $(41 \%)$. Adverse events with possible or probable relation to nimotuzumab included headache and vomiting in the same patient, and chills, mucositis, somnolence and vasovagal reaction in one patient each. Other events, such as convulsions, endocranial hypertension and bone pain, occurred in two patients, but it was considered unlikely that these events were related to the product. All events were classified as CTCAE grade 1 or 2.[35]

Abnormal laboratory test results, such as increased pyruvic and oxalacetic transaminases, were reported in three patients. Hepatitis $B$ was confirmed in one, and treatment with nimotuzumab was temporarily suspended for 6 months until that patient's hepatic profile returned to normal. In the other two patients, the events were of slight intensity, recovery was complete, and treatment was not interrupted. None of these metabolic alterations were related to nimotuzumab, although they occurred in patients receiving the antibody concurrently with chemotherapy.

Severe adverse events included hospitalization of one patient for surgery, and death of two patients due to rapid progression of the disease. None of these events was considered related to nimotuzumab.

Clinical Response and Survival Analysis Disease control with prolonged stabilization ( $>6$ months) was achieved in $64 \%$ of patients (14/22). Unexpectedly, three patients receiving nimotuzumab as monotherapy achieved complete response: one patient with a brain stem tumor, one with an ependymoblastoma and one with anaplastic astrocytoma. These objective responses were revealed during the maintenance phase, months after nimotuzumab treatment was initiated.

The survival rate was $82 \%$ at 6 months and $64 \%$ at 1 year; mean survival was 20.3 months (range 14.9-25.8 months) and median survival 19 months. Patients with brain stem tumors and low-grade glioma had the best survival rates, without reaching the median, since only 1 patient in each of these subgroups died; 5 of the 6 patients with brain stem tumors, and 3 of the 4 patients with low-grade glioma, were alive after more than 1 year of follow-up. An analysis of survival associated with the disease control variable showed that in patients with controlled disease $(n=14)$, mean survival was 28.6 months (range 24.2-33.1 months) without reaching the median (Figure 1), whereas, in patients who did not attain disease control $(n=8)$, mean survival was 6.3 months (range 2.3-9.7 months), and median survival was 5.7 months (range 0-12.4 months) (Figure 2).

The majority of patients who attained control of the disease also showed notable clinical improvement compared to their condition before treatment. The 3 patients with brain stem tumors and a KPS score of 50 associated with cranial nerve paralysis (VI, VII, VIII, $\mathrm{IX}$ and $\mathrm{X}$ cranial pairs), one of whom used a gastrostomy tube, recovered these neurological functions during the course of nimotuzumab treatment, including the digestive function. In the remaining patients with controlled disease, whose neurological impairment was less severe (presenting typical symptoms of disease progression before treatment, such as asthenia, headache and convulsions), the intensity and frequency of these symptoms gradually diminished and finally disappeared. As a result, these patients were able to resume normal daily life and even attend school or a child care center. 
Figure 1: Survival Functions in Total Patient Population $(n=22)$

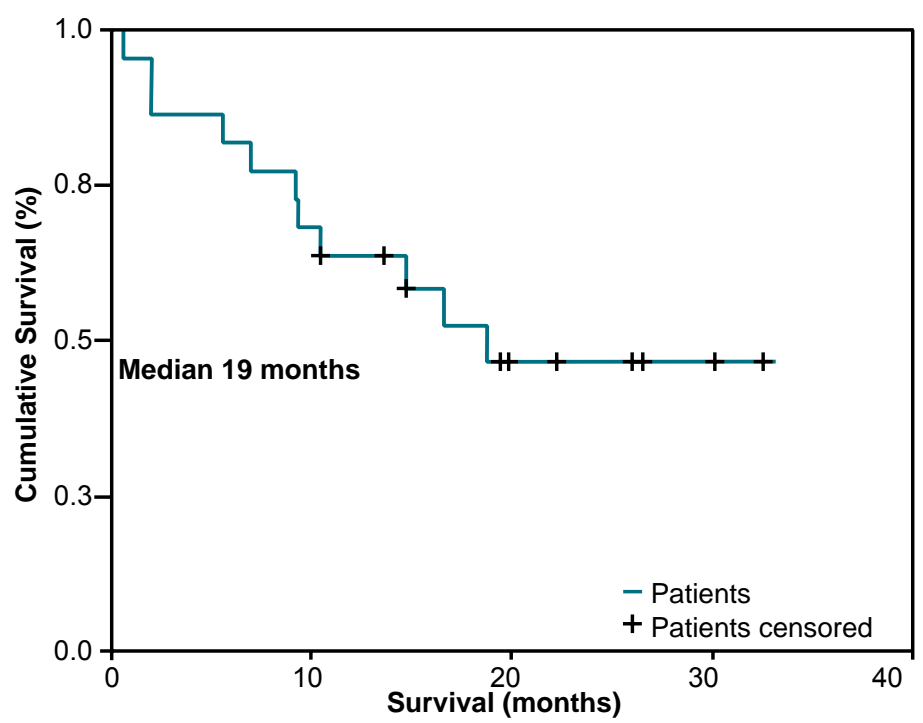

Figure 2: Survival Functions by Clinical Evolution

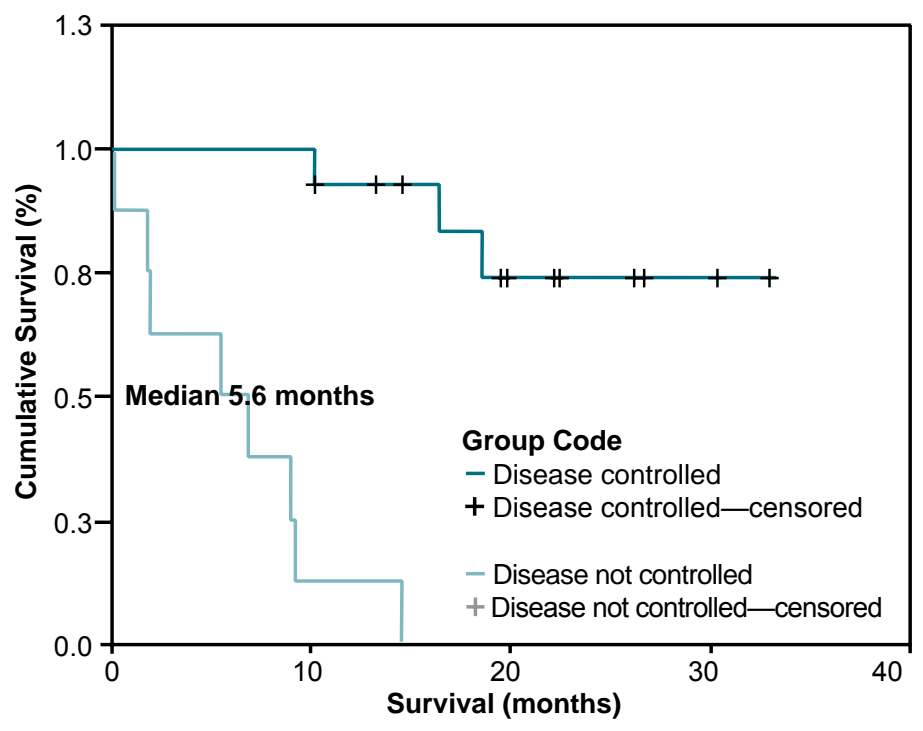

Disease controlled: $n=14$; Disease not controlled: $n=8$; Logrank $p=0.000$

\section{DISCUSSION}

This is the first report in Cuba on the feasibility of using nimotuzumab in a pediatric population with progressive or recurrent brain tumors, described in terms of the product's safety and therapeutic benefits, with a particular focus on the clinical management of this new product in an expanded access program.

The safety profile describes events similar in type, intensity and frequency to those previously reported with nimotuzumab, especially in clinical trials with pediatric brain tumor patients.[21,22]

An important feature of this product observed in the present study was the maintenance of repeated doses over a prolonged period of time-more than 1 year of treatmentwhich were very well tolerated. This was also observed when nimotuzumab was combined concurrently with radiotherapy and/or chemotherapy, adding no greater toxicity, facilitating adherence to treatment, especially with chemotherapy. This therapeutic modality had not been previously explored in pediatric patients.

Neither skin toxicity nor diarrhea-events infrequently associated with nimotuzumab-were reported, reaffirming a distinctive and favorable feature of its safety profile compared to other anti-EGFR products, for which skin lesions and diarrhea-sometimes very severe-are reported in $>70 \%$ of patients exposed.[37]

Although evaluating antitumor response was not the main objective of the study, unexpected objective responses were found (one partial response and three complete responses). Three of these patients received nimotuzumab as monotherapy, confirming the direct antitumor activity of the product. This activity was previously described in a phase II trial that included pediatric patients with recurrent glial tumors, with a $15.8 \%$ objective response rate (three partial responses in 19 patients), predominantly in patients with brain stem tumors.[21]

Non-clinical experiments with nimotuzumab show that this antibody intervenes in proapoptotic, antiproliferative and antiangiogenic mechanisms,[38] which may explain the clinically observed antitumor response.

Attainment of controlled disease in $64 \%$ of the 22 patients treated was especially encouraging. Prolonged radiological stabilization, without compromising the patient's general status, and gradual improvement in neurological functions constituted decisive medical criteria for continuing nimotuzumab treatment.

Most of the patients who attained stabilization and control of their disease survived over 1 year, according to patient follow-up. These variables (disease control and survival) were also associated in the study mentioned above,[21] in which 15 of 19 patients with brain stem tumors treated with nimotuzumab monotherapy were followed-up until their death $(78.9 \%)$, resulting in a median survival of 116 days (3.8 months) in a range of 73-284 days.[21] In our study, this survival benefit was extended to other histological types, such as ependymoblastoma and low-grade glioma.

Our results suggest that the use of nimotuzumab in progressive or recurrent pediatric brain tumors-which have a very bad prognosis per se-provides prolonged stabilization. These findings also demonstrate its capacity to modify the natural evolution of these tumors-even if the monoclonal antibody does not produce significant tumor reduction-increasing survival from barely a few weeks, as reported in the literature,[11,12] to several months, even over 1 year, mainly in patients with brain stem tumors and low-grade gliomas.

Recent studies using biological products targeting tumors, such as bevacizumab, trastuzumab and rituximab, have recommended that disease stabilization achieved with these therapies-or even early progression, with no deterioration in the patient's functional status or evidence of severe toxicity-not be considered criterion for interrupting treatment. On the contrary, if treatment is allowed to continue, it may result in clinical benefits in terms of prolonged survival and improved quality of life.[39-41]

These observations, which emphasize maintaining treatment beyond an objective antitumor response, are also applicable to 
nimotuzumab. Thus a new clinical response paradigm, distinct from that used to manage chemotherapy and radiotherapy, is reinforced, precisely because of the specificity of these biological products for the tumors they target, and their generally lower toxicity.

Due to the limited number of patients in the sample studied, the heterogeneity of the tumors and variety of regimens used, definitive conclusions about the clinical benefit of this product cannot be made. However, we consider the findings of this study quite encouraging for pediatric patients with progressive or recurrent brain tumors, a malignant disease with a very low incidence in Cuba but also with very limited therapeutic options and insufficient therapeutic efficacy in terms of survival.

\section{CONCLUSIONS}

Nimotuzumab can be considered a new therapeutic option for pediatric patients with progressive or recurrent primary brain tumors. Its safety profile enables maintained administration, inducing prolonged disease control, increased survival, reduced symptoms and improved neurological functions. Future trials may confirm these results. -1 -

\section{REFERENCES}

1. Strother DR, Pollack IF. Tumors of the Central Nervous System. In: Pizzo PA, Poplack DG, editors. Principles and Practice of Pediatric Oncology. Philadelphia: Lippincot Williams and Wilkins; 2002. p. 751-814.

2. Lanskoswky P. Manual of Pediatric Hematology Oncology. San Diego: Academic Press; 2000. p. 471-91.

3. Wilne S, Collier J, Kennedy C, Koler K, Grundy $\mathrm{R}$, Walker D. Presentation of childhood CNS tumours: a systematic review and meta-analysis. Lancet Oncol. 2007 Aug;8(8):685-95.

4. Taquechel $\mathrm{CO}$, Corral $\mathrm{N}$, Verdecia $\mathrm{M}$, Legrá $\mathrm{R}$, Psudides A. Tumores del sistema nervioso central en la infancia. MEDISAN. 2003;7(4):30-4.

5. Packer RJ. CNS tumors. In: Agarwal B, Perilongo G, Calaminus G, Eden T, editors. SIOP Education Book; 2007

6. Jahraus CD, Tarbell NJ. Optic Pathway Gliomas. Pediatr Blood Cancer. 2006 May 1; 46(5):58696. Review.

7. Broniscer A, Gajjar A. Supratentorial High Grade Astrocitomas and Difusse Brain Stem Glioma: Two Challenges for the Pediatric Oncologist. Oncologist. 2004;9(2):197-206.

8. Macdonald TJ, Rood BR, Santi MR, Vezina G, Bingaman $\mathrm{K}$, Cohen $\mathrm{PH}$, et al. Advances in the Diagnosis, Molecular Genetics and Treatment of Pediatric Embryonal CNS Tumors. Oncologist. 2003;8(2):174-86

9. Packer RJ, Gurney JG, Punyko JA, Donaldson SS, Inskip PD, Stovall M, et al. Long term neurologic and neurosensory sequelae in adult survivors of a childhood brain tumor: childhood cancer survivor study. J Clin Oncol. 2003 Sep 1;21(7):3255-61.

10. Finlay $\mathrm{JL}$, Zacharoulis $\mathrm{S}$. The treatment of high grade gliomas and diffuse intrinsic pontine tumors of childhood and adolescence: a historical and futuristic perspective. J Neurooncol. 2005 Dec;75(3):253-66.

11. Bowers DC, Gargan L, Weprin BE, Mulne AF, Elterman RD, Munoz L, et al. Impact of site of tumor recurrence upon survival for children with recurrent or progressive medulloblastoma. J Neurosurg. 2007 Jul;107(1 Suppl):5-10.

12. Packer Roger J. Progress and challenges in childhood brain tumors. J Neurooncol. 2005 Dec;75(3):239-42.

13. Warren KE. Molecularly targeted therapy for pediatric brain tumors. J Neurooncol. 2005 Dec;75(3):335-43.

14. Basel J. Targeting tyrosine kinases in cancer: the second wave. Science. 2006 May 26;312(5777):1175-8.

15. Mateo C, Moreno E, Amour K, Lombardero J, Harris W, Pérez R. Humanization of a mouse monoclonal antibody that blocks the Epidermal growth factor receptor: Recovery of antagonistic activity. Immunotechnology. 1997 May;3(1):71-81.

16. World Health Organization Drug Information. International Nonproprietary Names for Pharmaceutical Substances (INN). Proposed INN: List 94. 2005;19(4):333.

17. Crombet $T$, Torres $O$, Rodríguez $V$, Menéndez A, Stevenson A, Ramos M, et al. Phase I clinical evaluation of a neutralizing monoclonal antibody against Epidermal Growth Factor Receptor in advanced brain tumor patients. Hybridoma. 2001;20(2):131-36.

18. Crombet T, Torres L, Neninger E, Catala M, Solano ME, Perera A, et al. Pharmacological evaluation of humanized anti-epidermal growth factor receptor, monoclonal antibody $h-R 3$, in patients with advanced epithelial-derived cancer. J Immunother. $2003 \mathrm{Apr} ; 26(2): 139-48$.

19. Crombet $T$, Osorio M, Cruz T, Roca C, Del Castillo R, Mon R, et al. Use of the humanized anti-epidermal growth factor receptor monoclonal antibody h-R3 in combination with radiotherapy in the treatment of locally advanced head and neck cancer patients. J Clin Oncol. 2004;22(9):1646-54.

20. Crombet T, Figueredo J, Catalá M, González S, Selva JC, Cruz TM, et al. Treatment of highgrade glioma patients with the humanized antiepidermal growth factor receptor (EGFR) antibody h-R3: report from a phase I/II trial. Cancer Biol Ther. 2006;5(4):375-9.

21. Bode U, Buchen S, Warmuth-Metz M, Pietsch T, Bach F, Fleischhack G. Final report of a phase II trial of nimotuzumab in the treatment of refractory and relapsed high-grade gliomas in children and adolescents. J Clin Oncol. 2007;25(18 Suppl):S2006.

22. Bode U, Windelberg M, Massimino M, Khuhlaeva E, Warmuth-Metz M, Kortmann R, et al. Phase III trial of nimotuzumab for the treatment of newly diagnosed diffuse intrinsic pontine gliomas in children and adolescents. J Clin Oncol. 2008;26(15 Suppl):S2058.

23. Brade AM, Siu L, Oza AM, Southwood B, De Borja M, Pond G, et al. A phase I study of the humanized anti-epidermal growth factor receptor (EGFR) monoclonal antibody (mAb) TheraCIM-h-R3 (Nimotuzumab) in patients with advanced solid tumors. J Clin Oncol. 2006;24(18 Suppl):S13054.

24. Bebb DG, Brade AM, Smith C, Rorke S, Sherman I. Preliminary results of an escalating dose phase I clinical trial of the anti-EGFR monoclonal antibody nimotuzumab in combination with external radiotherapy in patients diagnosed with stage $\mathrm{Ilb}$, III or IV non-small cell lung cancer unsuitable for radical therapy. J Clin Oncol. 2008;26(15 Suppl):S3037.
25. Xiao-Dong $\mathrm{H}$, Guo-Zhen $\mathrm{X}$, Lil $\mathrm{G}$, Jun-Lin $\mathrm{Y}$, Tai-Xiang L, Shao-Xiong W, et al. Multi-center Phase II Clinical Trial of h-R3, the Recombined Humanized Anti-Epidermal Factor Receptor Monoclonal Antibody, in Combination with Radiotherapy in Patients with Locoregionally Advanced Nasopharyngeal Carcinoma. Chin J Oncol. 2007;29(3):197-201.

26. ClinicalTrials.gov [homepage on the Internet]. YM Biosciences;[updated 2009 June 3; cited 2009 Feb]. Phase 2 Study of Nimotuzumab in Pediatric Recurrent Diffuse Intrinsic Pontine Glioma. Available from: http://www.clinicaltrial.gov/ ct2/show/NCT00600054.

27. Ramakrishnan MS, Eswariah A, Crombet T, Piedra P, Saurez G, lyer H, et al. Nimotuzumab, a promising therapeutic monoclonal for treatment of tumors of epithelial origin. mAbs. 2009 JanFeb;1(1):41-8.

28. Reddy BK, Vidyasagar M, Shenoy K, Shenoy A, Viswanath L, Babu G, et al. BIOMAb EGFRTM (Nimotuzumab/h-r3) in Combination with standard of care in squamous cell carcinoma of head and neck (SCCHN). Int J Radiat Oncol Biol Phys. 2007;69(3 Suppl):S450.

29. Pendharkar D, Gupta S, Pal MK, Hakim S, Rashid T. Feasibility of combining humanized anti-epidermal growth factor receptor monoclonal antibody h-R3 (nimotuzumab) with chemotherapy-A study of toxicity profile and tolerance. J Clin Oncol. 2007;25(18 Suppl):S14151.

30. Bebb DG, Brade AM, Smith C, Rorke S, Sherman I. Preliminary results of an escalating dose phase I clinical trial of the anti-EGFR monoclonal antibody nimotuzumab in combination with external radiotherapy in patients diagnosed with stage IIb, III or IV nonsmall cell lung cancer unsuitable for radical therapy. J Clin Oncol. 2008 May;26 (15 Suppl):S3037.

31. ClinicalTrials.gov [homepage on the Internet]. YM Biosciences;[updated 2009 Jan 15; cited 2009 Jan 15]. Iressa expanded access Program program. Available from: http://clinicaltrials.gov/ show/NCT00034879

32. Okie S. Access before Approval- A Right to Take Experimental Drugs? N Engl J Med. 2006 Aug 3;355(5):437-40.

33. Guideline on compassionate use of medicinal products, pursuant to article 83 of regulation $(\mathrm{ec})$ no 726/2004. London, 19 July 2007. Doc. Ref: EMEA/27170/2006.

34. Ünal E, Sahdev I. Use of gemtuzumab ozogamicin in the treatment of pediatric relapsed/refractory Acute Myeloid Leukemia. Turk J Hematol. 2008 Mar;25(1):36-41.

35. National Cancer Institute [Internet]. Rockville (MD): Cancer Therapy Evaluation Program, Common Terminology Criteria for Adverse Events, Version 3.0, DCTD, $\mathrm{NCl}, \mathrm{NIH}, \mathrm{DHHS}$ 
(US); [updated 2008 May 29; cited 2003 Mar 31]. Available from: http://ctep.cancer.gov.

36. Zeltzer PM, Friedman HS, Norris DG, Ragab AH. Criteria and definition of response and relapse in children with brain tumor. Cancer. 1985;56(7 Suppl):S1824-6.

37. Ciardiello F, Tortora G. Anti-epidermal growth factor receptor drugs in cancer therapy. Expert Opin Investing Drugs. 2002 Jun;11(6):755-68.

38. Crombet T, Rak J, Pérez R, Viloria-Petit A. Antiproliferative, anti-angiogenic and proapoptotic activity of h-R3: A Humanized anti-EGFR antibody. Int J Cancer. 2002 Oct 20;101(6):567-75.

39. Grothey A, Sugrue MM, Purdie DM, Dong W, Sargent D, Hedrick E, et al. Bevacizumab Beyond First Progression Is Associated With Prolonged Overall Survival in Metastatic Colorectal Cancer: Results From a Large Observational Cohort Study (BRiTE). J Clin Oncol. 2008 Nov 20;26(33):5326-34.

40. Metro G, Mottolese M, Fabi A. HER-2-positive metastatic breast cancer: trastuzumab and beyond. Expert Opin Pharmacother. 2008 Oct;9(15):2583-601.

41. Hainsworth JD, Litchy S, Shaffer DW, Lackey VL, Grimaldi M, Greco FA. Maximizing Therapeu- tic Benefit of Rituximab: Maintenance Therapy Versus Re-Treatment at Progression in Patients With Indolent Non-Hodgkin's LymphomaA Randomized Phase II Trial of the Minnie Pearl Cancer Research Network. J Clin Oncol. 2005;23(6):1088-95

\section{THE AUTHORS}

Giselle Saurez Martínez (Corresponding Author: giselle@cim.sld.cu), specialist in family medicine, Molecular Immunology Center (CIM), Havana, Cuba.

Ricardo Cabanas Armada, pediatrician and oncologist, Juan Manuel Márquez Pediatric Teaching Hospital, Havana, Cuba.

Melba Zaldívar Santiesteban, neurosurgeon, Octavio de la Concepción y de la Pedraja Pediatric Hospital, Holguín, Cuba.

Tania Garnier Ávila, neurobiologist, Octavio de la Concepción y de la Pedraja Pediatric Hospital, Holguín, Cuba.
Bárbara Iglesias Castillo, oncologist, Pepe Portilla Pediatric Hospital, Pinar del Río, Cuba.

Patricia Piedra Sierra, pharmacologist, Molecular Immunology Center, Havana, Cuba.

Martha Ríos Castillo, neurosurgeon, Juan Manuel Márquez Pediatric Teaching Hospital, Havana, Cuba.

Martha Longchong Ramos, oncologist, Juan Manuel Márquez Pediatric Teaching Hospital, Havana, Cuba.

Normando Iznaga Escobar, nuclear physicist and pharmacologist, Molecular Immunology Center, Havana.

Agustín Lage Dávila, biochemist, Director, Molecular Immunology Center, Havana.

Submitted: January 9, 2009

Approved for publication: June 1, 2009 